\title{
Cusp-Shaped Elastic Creases and Furrows
}

\author{
S. Karpitschka, ${ }^{1 *}$ J. Eggers, ${ }^{2}$ A. Pandey, ${ }^{1}$ and J. H. Snoeijer ${ }^{1,3}$ \\ ${ }^{1}$ Physics of Fluids Group, Faculty of Science and Technology, Mesa+ Institute, University of Twente, \\ 7500 AE Enschede, Netherlands \\ ${ }^{2}$ School of Mathematics, University of Bristol, University Walk, Bristol BS8 1 TW, United Kingdom \\ ${ }^{3}$ Mesoscopic Transport Phenomena, Eindhoven University of Technology, Den Dolech 2, 5612 AZ Eindhoven, Netherlands
}

(Received 3 May 2017; revised manuscript received 8 September 2017; published 7 November 2017)

The surfaces of growing biological tissues, swelling gels, and compressed rubbers do not remain smooth, but frequently exhibit highly localized inward folds. We reveal the morphology of this surface folding in a novel experimental setup, which permits us to deform the surface of a soft gel in a controlled fashion. The interface first forms a sharp furrow, whose tip size decreases rapidly with deformation. Above a critical deformation, the furrow bifurcates to an inward folded crease of vanishing tip size. We show experimentally and numerically that both creases and furrows exhibit a universal cusp shape, whose width scales like $y^{3 / 2}$ at a distance $y$ from the tip. We provide a similarity theory that captures the singular profiles before and after the self-folding bifurcation, and derive the length of the fold from finite deformation elasticity.

DOI: 10.1103/PhysRevLett.119.198001

Compressing a slice of soft white bread, one observes the formation of a crease, a localized indentation where part of the surface folds into a self-contact. Similar patterns appear, for instance, on the surfaces of swelling gels $[1,2]$. In biology, such elastic structures are called sulci, which are prime morphological features of human brains and growing tumors [3-5]. As a result, creases have attracted considerable attention, experimentally, theoretically, and from a numerical point of view [2-18]. Yet in spite of their ubiquity and importance, a quantitative theoretical description of the morphology of localized indentations is still missing.

Past approaches have focused on the idealized problem of a half-space of elastic material, which is compressed uniformly parallel to the interface. Above a critical compression, the uniform state becomes unstable toward sinusoidal deformation of the interface [19]. However, since this setup lacks a characteristic length scale, perturbations grow without bound even in the nonlinear regime. Additional regularizing features have to be invoked, such as adding a thin film of stiff material on the surface $[3,5]$. While the metastability of smooth and creased configurations has been studied in some detail $[3,8,14]$, much less is known on the profiles of localized indentations. Here, we propose a new experimental setup that guarantees the formation of a single indentation of finite size, which bifurcates between two different structures, see Fig. 1. It allows us to reveal for the first time the self-similar shape

Published by the American Physical Society under the terms of the Creative Commons Attribution 4.0 International license. Further distribution of this work must maintain attribution to the author(s) and the published article's title, journal citation, and DOI. properties of both structures, and to provide quantitative analytical descriptions thereof.

A highly deformable Polydimethylsiloxane gel (Dow Corning CY52-276, components $A$ and $B$ mixed 1:1, shear modulus $\mu=1 \mathrm{kPa}$ ) is prepared in a container of footprint $3 \times 3 \mathrm{~cm}$. A 10 times stiffer gel was prepared for some experiments by adding 5\% of Dow Corning Sylgard 184 (polymer and curing agent mixed 10:1, yielding $\mu=11 \mathrm{kPa})$. The gels were cured overnight at room temperature, protecting the air-exposed free surface from dust. By depositing a water drop inside the gel prior to curing, we create a liquid inclusion of initial radius $R$ at the bottom of the container. $l$ denotes the initial distance between inclusion and free surface (cf. Fig. 1); we used $\ell=1-8 \mathrm{~mm}$ and $R / \ell=0.4-6$. Subsequently, the water is extracted slowly ( $\gtrsim 100 \mathrm{~s}$ for the droplet volume) through a small hole at the bottom (cf. Fig. 1), creating a

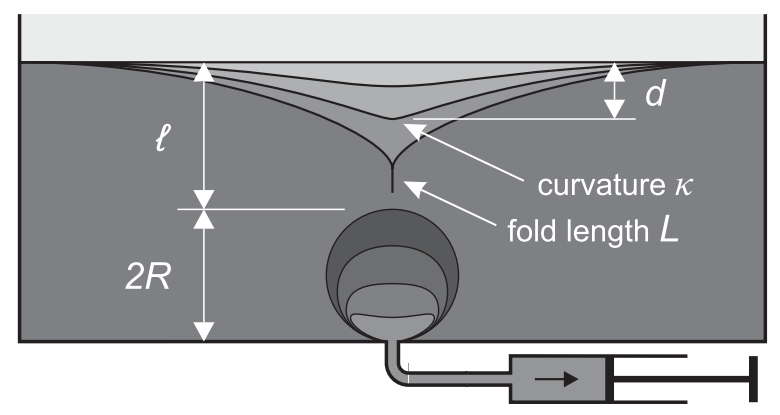

FIG. 1. The interface of a soft gel is deformed by slowly reducing the volume of a liquid inclusion (initial radius $R$ ), located at an initial distance $\ell$ below the free surface. First, a localized furrow with tip curvature $\kappa$ forms; at larger deformations the furrow bifurcates into a crease that folds the free surface onto itself over a length $L$. 
quasistationary axisymmetric strain field in the gel, and an increasingly sharp indentation of the free surface. A twodimensional version of the experiment was realized by creating a cylindrical inclusion at the bottom of the container. In that case, the inclusion was templated by a cylinder of polyethylene glycol $\left(M=1000 \mathrm{~g} / \mathrm{mol}\right.$, melting point $37^{\circ}-$ $40^{\circ}$ ), which was removed by melting after the gel was cured.

The free surface profile was measured as a shadowgraph through a long-distance microscope with a spatial resolution on the order of $10 \mu \mathrm{m}$. The deformation field and the length of the self-contacting surface fold were determined by tracking fluorescent particles embedded inside the gel (see the Supplemental Material for details [20]). The amplitude of the deformation is measured by $d$, the deflection of the free surface relative to its reference level. The sharpness of the deflection is quantified by its curvature $\kappa$ in the image plane.

Figure 2 shows the result of a typical experimental run, obtained by first deflating the water drop to vanishing size (filled symbols), and subsequently reinflating it up again to its original size (open symbols). The deformation is quantified by $d / \ell$ shown on the horizontal axis. Upon increasing deformation, the gel develops an increasingly sharp furrow, as measured by the dimensionless radius of curvature $(\kappa \ell)^{-1}$ [Fig. 2(a); the furrow's self-similar shape

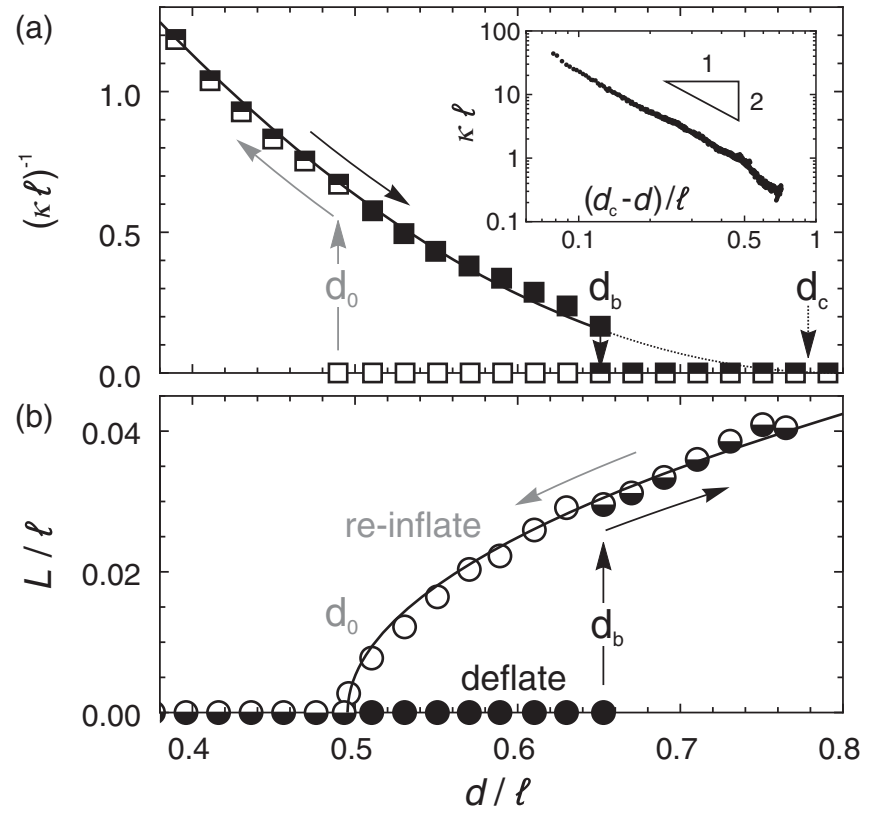

FIG. 2. Bifurcation between the "furrow" and the "crease" (axisymmetric cavity, $\mu=1 \mathrm{kPa}, R=2.1 \mathrm{~mm}, \ell=2.4 \mathrm{~mm}$ ). (a) Tip radius of curvature $\kappa^{-1}$ in the image plane and (b) fold length $L$, as a function of the deformation amplitude $d$. Arrows indicate the course of the experiment. Increasing the deformation (filled symbols) beyond $d_{b}$ nucleates a fold of finite length. Decreasing the deformation again (open symbols), the fold disappears continuously at $d_{0}$. The solid lines represent Eqs. (1) and (2) for the curvature and the fold length, respectively. The inset demonstrates the scaling law (1) in a double logarithmic plot (here, $R=1.9 \mathrm{~mm}, \ell=2.4 \mathrm{~mm}$ ). is investigated below]. At a deformation $d=d_{b}$, the furrow bifurcates toward a crease (similar to previously reported behavior [3,14]): part of the surface folds into a self-contact of length $L$, connected to a free-surface cusp of vanishing tip curvature. In the axisymmetric version of the experiment, this is accompanied by a breaking of axisymmetry, the crease being essentially two dimensional. Experimental data are fitted with the scaling law [Fig. 2(a), solid line]

$$
\kappa=k \ell\left(d_{c}-d\right)^{-2},
$$

which suggests the existence of a critical deformation $d_{c}$ at which the tip radius of curvature vanishes; however, this critical scaling is cut off by a discontinuous (first order) transition toward the crease at $d=d_{b}$.

Directly after formation of the crease, a self-contact of length $L \approx 0.03 \ell$ forms, while the radius of curvature of the new structure jumps to zero. With increasing deformation, $L$ increases further (circles). Reinflating the liquid inclusion again, so as to decrease $d, L$ decreases beyond its original value to go to zero in a continuous fashion at another critical value $d_{0}$; this is described by the critical behavior [Fig. 2(b), solid line]

$$
L=c \sqrt{\ell\left(d-d_{0}\right)}
$$

reminiscent of a second order transition, to be discussed below. Below $d=d_{0}$, the crease disappears and the interface shape returns to a furrow, in the course of which the tip radius jumps to a finite value. Decreasing the deformation further, the tip radius returns to its original value along the same curve, indicating that the entire process is reversible. To check reversibility, we repeated the whole cycle several times for each specimen, which yielded nearly identical results (typical deviation $\lesssim 2 \%$ ). Merely, $d_{b}$ was slightly smaller by about $4 \%$ as compared to the first creasing event. This could indicate the formation of a localized nucleation seed due to the initial creasing [15].

The scaling laws (1) and (2) are universal features of the creasing instability. These were consistently observed, where we in total considered about 25 different configurations (axisymmetric and 2D, soft and stiff gels) with $\ell=1-8 \mathrm{~mm}$ and $R / \ell=0.4-6 . R \ll \ell$ precludes large deformations because of the limited droplet volume. For $R \lesssim 0.8 \ell$, creasing was not induced before the droplet was drained completely (axisymmetric samples, $R \lesssim 0.5 \ell$ for 2D). Otherwise, all experiments show similar curves as in Fig. 2 with universal scaling laws. As expected, the precise values of $d_{b}$ and $d_{0}$ are not universal (see the Supplemental Material [20]).

The shape of the furrow is perfectly self-similar, as is shown in Fig. 3. To describe this self-similar structure analytically, we hypothesize that the surface shape is described by a plane curve with a smooth parametric representation $x(s), y(s)$, which has been used successfully 

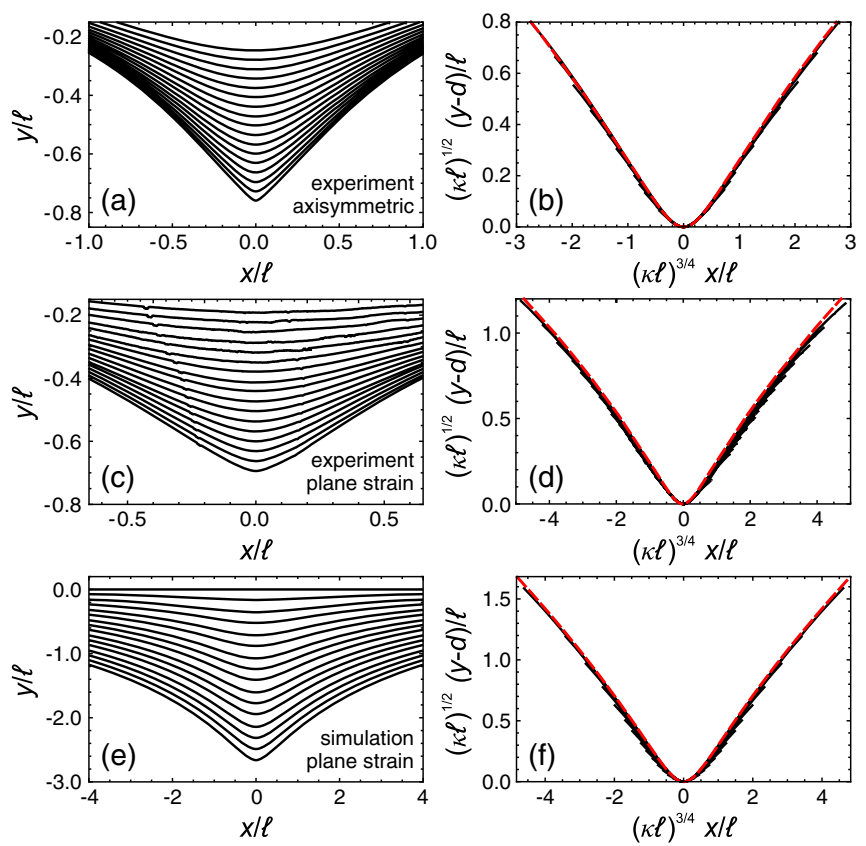

FIG. 3. Self-similar evolution of free surface profiles for 3D and $2 \mathrm{D}$ experiments, and 2D simulations, prior to the creasing instability. Left: measured (simulated) profiles. Right: profiles rescaled according to Eq. (4), and superimposed with the similarity solution $\Phi$ (red).

to describe cusp formation quantitatively in free surface flows and in optics [21,22]; a rigorous rationale for such a description is provided for example by a complex mapping between the free surface and the unit circle [23], as has been verified experimentally for viscous flows [24]. To picture cusp formation of a parametrized curve $x(s), y(s)$ geometrically, one can imagine these components being deformed smoothly such that the curve self-intersects. At the point of self-intersection, the curve is a cusp with a singular tip. Just before intersection the curve opens into a universal smooth curve.

Namely, a critical point of the curve corresponds to $x^{\prime}(0)=y^{\prime}(0)=0$, so expanding about $s=0$ to lowest nontrivial order yields [25]

$$
x=\epsilon s+s^{3} /\left(2^{3 / 2} a\right), \quad y=s^{2} / 2,
$$

where $\epsilon=0$ corresponds to the critical (cusp) point, and $a$ is a parameter controlling the opening of the cusp $y=(a x)^{2 / 3}$. In the $x$ component we expanded to third order, since any quadratic term can be eliminated using $y$, implying a rotation. The curvature of Eq. (3) at the origin is $\kappa=\epsilon^{-2}$, so Eq. (3) can be written in similarity form

$$
y \kappa^{1 / 2}=\Phi(\xi), \quad \xi=x \kappa^{3 / 4},
$$

where $\Phi$ is defined implicitly: $\xi^{2}=2 \Phi(1+\Phi /(\sqrt{2} a))^{2}$, see Ref. [25]. As seen in Figs. 3(b), 3(d), and 3(f), the similarity form (4) is in excellent agreement with both $2 \mathrm{D}$ and 3D experiments and simulation, and the collapsed data agrees very well with the universal similarity function $\Phi(\xi)$. The single adjustable parameter $a$ is determined by the outer geometry of the problem. In addition, the relation between the vertical deformation scale and $\kappa$ implied by Eq. (4) is consistent with Eq. (1). Of course, this geometric analysis cannot describe the precise value of the tip curvature $\kappa(d)$, which must be derived from finite deformation elasticity theory $[26,27]$.

To show that the observations above are well described by the mechanics of elasticity, we performed twodimensional (plane strain) finite element simulations implemented in oomph-lib, an object-oriented, open-source finite-element library for the simulation of physics problems [28], using the theory of finite deformations [26], with an incompressible neo-Hookean constitutive equation [27]. In finite deformation theory the coordinates $\mathbf{X}$ of the undeformed state of the system (the reference state, see Fig. 4) are mapped upon the current, deformed state of the system as $\mathbf{x}=\mathbf{f}(\mathbf{X})$. For a neo-Hookean elastic material, the Cauchy stress is [26]

$$
\sigma_{i j}=\mu \frac{\partial x_{i}}{\partial X_{k}} \frac{\partial x_{j}}{\partial X_{k}}-p \delta_{i j}
$$
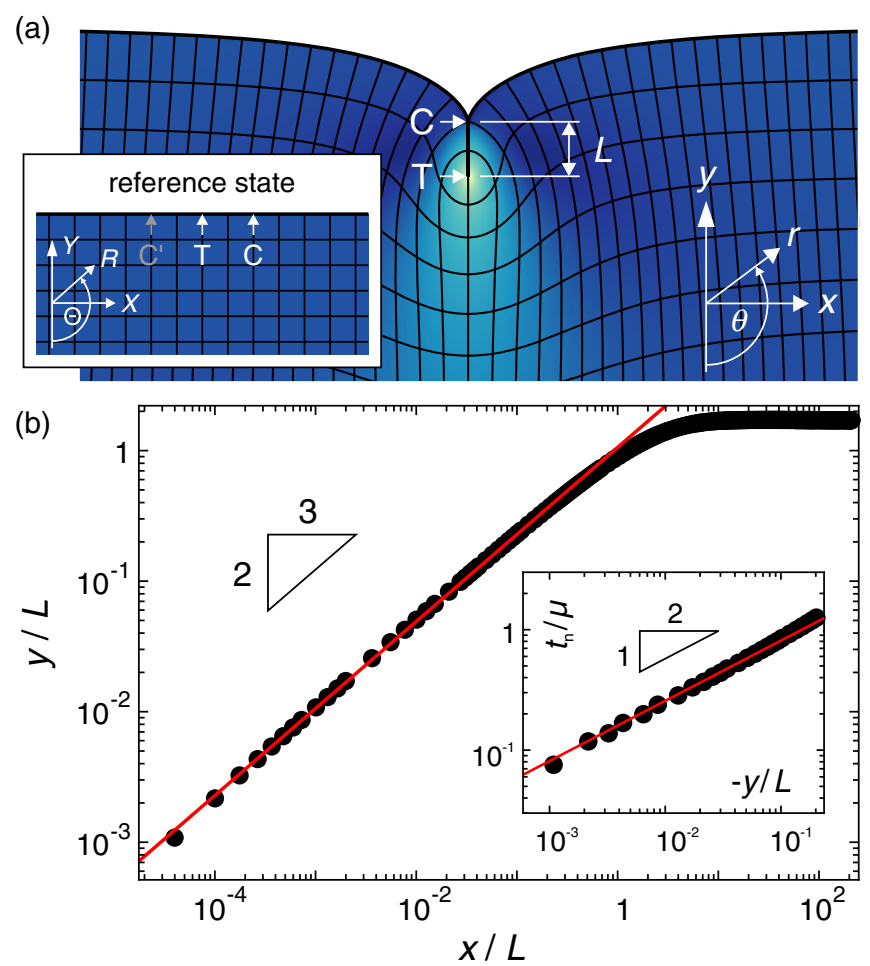

FIG. 4. Numerical simulation of the creased state, with a selfcontacting fold of length $L$; the tip of the fold is at $T$; the self-contact ends at $C$ (the tip of the cusp). (a) The deformed state $\mathbf{x}$, showing the solid pressure as a color plot; the reference state $\mathbf{X}$ is shown in the inset. (b) Surface profile of the crease above the fold ( $x$ and $y$ measured relative to $C$; red line, fit with a 2/3-power law). The inset shows the 1/2-power law of the normal (contact) traction near $C$. 
where $\mu$ is the shear modulus and $p$ the solid pressure (here defined up to a constant), which ensures incompressibility: $\operatorname{det}\left(\partial x_{i} / \partial X_{j}\right)=1$. Elastic equilibrium is determined by $\partial \sigma_{i k} / \partial x_{k}=0$. For simulation details see the Supplemental Material [20]. The result of the simulations is given in Figs. 3(e) and 3(f). They recover the same features as in experiments, including the similarity collapse with the same universal shape superimposed.

The remaining challenge is to understand the morphology of the creased state, which contains two singular points, respectively indicated as $C$ and $T$ in Fig. 4(a). To derive a solution of the creased state, we start from the fold solution around point $T[8,29]$, which maps an elastic halfspace onto a fold of infinite length [coordinates are defined in Fig. 4(a); here, the origin lies in point $T$ ]:

$$
\theta=2 \Theta, \quad r=R / \sqrt{2}, \quad p=-3 \mu \ln r / 2,
$$

which is an exact solution of Eq. (5). On the fold ( $x=0)$, the principal stretches are $\lambda_{x, y}=\sqrt{2}, 1 / \sqrt{2}$, and the elastic free energy density is $W=5 \mu / 4$. The logarithmic divergence of $p$ near $T$ is uncritical for a macroscopic description of the experiment since $p \lesssim 100 \mathrm{kPa}$ down to molecular length scales. To numerically simulate the creased state, we use a large domain $(\sim 320 L)$ under horizontal compression, impose Eq. (6) near $T$, and require a non-negative normal traction and vanishing tangential traction on $T C$ (see the Supplemental Material for details [20]).

Figure 4(a) shows the deformed computational domain, while Fig. 4(b) reveals a power-law behavior, $y \sim(a x)^{2 / 3}$, of the interface above the self-contact, with four decades of spatial resolution. Hence, the interface forms an ideal cusp, $y \sim x^{2 / 3}$, which is the limiting case $\epsilon=0$ in Eq. (3). In the Supplemental Material [20] we also provide experimental evidence for this scaling. To access the surface profile near the self-contact experimentally with sufficient spatial resolution, we bent an elastic rod until it creased on its surface and recorded its shadowgraph. Despite the significantly different outer geometry, we find the same $2 / 3$ exponent for the morphology of the crease, highlighting the universality of this result.

This scaling can be derived analytically by noting that near $C$, where the fold opens, the shape is slender: $x \ll y$ (from now on we use $C$ as the coordinate origin). Hence, deformations relative to that of the fold are small and we can expand to linear order in the deformations $u, v$ :

$x=\lambda X+u(\lambda X, Y / \lambda), \quad y=\lambda^{-1} Y+v(X \lambda, Y / \lambda)$,

where $\lambda$ is the stretch near $C$. We use coordinate systems as shown in Fig. 4(a), but for simplicity use a reference state that is rotated clockwise by $90 \mathrm{deg}$. As shown in Ref. [19], if we introduce a stream function $u=\partial_{y} \psi, v=-\partial_{x} \psi$, the linearized elasticity problem reduces to

$$
\triangle \bar{\triangle} \psi \equiv \triangle \Psi=0,
$$

where $\triangle$ and $\bar{\triangle}$ denote the Laplacian in the deformed $(x, y)$ and reference $(X, Y)$ coordinates, respectively.

Similar to the analysis of the cusp in a viscous fluid [21], we make the self-similar ansatz $\psi=r^{\alpha} f(\theta)=R^{\alpha} \bar{f}(\Theta)$; $\theta=\Theta=\pi$ corresponds to the cusp line, along which we impose vanishing shear and, outside the self-contact, vanishing normal stress. Using that $f$ is odd, we find from the second equation (8) that [21]

$$
\bar{\triangle} \psi=\Psi=A r^{\alpha-2} \sin (\alpha-2) \theta
$$

and $p=A \mu r^{\alpha-2} \cos (\alpha-2) \theta$, where $A$ is an arbitrary constant. Now solving Eq. (9) in reference coordinates, homogeneous solutions are $\bar{f}_{\{1,2\}}=\{\sin , \cos \}(\alpha \Theta)$. In polar coordinates, the transformation between deformed and reference coordinates reads $R / r=\left(\lambda^{2} \cos ^{2}(\theta)+\lambda^{-2} \sin ^{2}(\theta)\right)^{1 / 2} \equiv g$ and $\lambda^{2} \tan \Theta=\tan \theta$, and so

$$
f_{\{1,2\}}=\{\sin , \cos \}(\alpha \Theta) g^{\alpha} .
$$

An odd particular solution of Eq. (9) is found from the standard formula as $f_{p}=A\left(f_{1} I_{2}(\theta)-f_{2} I_{1}(\theta)\right)$, where

$$
I_{\{1,2\}}(\theta)=\int_{0}^{\theta} \frac{\{\sin , \cos \}(\alpha \Theta) \sin (\alpha-2) \theta}{\alpha g^{\alpha}} d \theta .
$$

A general solution to Eq. (8) can be written $f=f_{p}+B f_{1}$, where the constants $A, B$ must be chosen to satisfy $\sigma_{i j} n_{j}=0$ at $\theta=\pi$, where $n_{j}$ is the true normal.

For there to be a nontrivial solution, the determinant of this system of equations must vanish, which after using that $2 \alpha(\alpha-1) I_{1}(\pi)=-\lambda^{2-\alpha} \sin (2 \pi \alpha) /\left(\lambda^{2}+1\right)$ yields the condition $\sin (2 \pi \alpha)=0$. Thus, the determinant vanishes for $\alpha=i / 2$, where $i=1,2,3, \ldots$, irrespective of the stretch $\lambda$ near $C$. Among these possible solutions, the dominant value of $\alpha$ for which the pressure is not singular at the cusp tip is $\alpha=5 / 2$, which means that the cusp opens with the universal exponent $u \propto y^{3 / 2}$, as is confirmed over four decades in Fig. 4(b). Accordingly, $\alpha=5 / 2$ implies that the normal traction near the edge of the contact scales like $t_{n} \propto|y|^{1 / 2}$, as confirmed numerically (inset). Thus, both deformation and traction scale in the same way as a Hertz contact [30]. We also note that the above calculation provides a rationale for the scaling in the far field of the furrow: away from its rounded tip, the furrow's geometry is again slender and can be described by the same analysis.

Finally, we analyze the length $L$ of the fold. Since the energy density of the fold solution (6) is constant, the contribution from the fold is $E_{f}=A_{0} L^{2}$, since $L$ sets the size of the area over which deformation is significant. We can assume that the energy $E_{0}(d)$ of the rest of the strain field is a smooth function of the deformation $d$. Hence, if the creation of the fold takes place in a reversible fashion, 
we have $A_{0} L^{2}+E_{0}(d)=$ const. Expanding $E_{0}$ linearly about $d=d_{0}$, where $L=0$, we obtain Eq. (2). Apart from the experiment of Fig. 2, this scaling law is confirmed with great precision by the numerical simulation of a neoHookean material shown in Fig. 4 [20].

In conclusion, our liquid-inclusion experiments allowed us to investigate quantitatively localized furrows and creases that form on the surface of an elastic medium under compression, and to document the hysteretic transitions between them. We are able to describe the self-similar shapes of these furrows quantitatively, in agreement with both experiment and neo-Hookean nonlinear elasticity. Based on elasticity theory, we are able to explain the $x \propto$ $y^{3 / 2}$ scaling of the width of both the furrow and the crease. These scaling laws reveal that the elastic singularity is a "true" geometric cusp, and belongs to the same universality class as caustics in optics and free surface flows.

We are grateful to J. Dervaux and L. van Wijngaarden for discussions. S. K., A.P., and J.H.S. acknowledge financial support from European Research Council (ERC) Consolidator Grant No. 616918. The work of J.E. was supported by Leverhulme Trust Research Project Grant No. RPG-2012-568.

*karpitschka@googlemail.com

[1] T. Tanaka, Physica A: Statistical Mechanics and its Applications 140, 261 (1986).

[2] J. K. V. Trujillo and R. C. Hayward, Soft Matter 4, 564 (2008).

[3] E. Hohlfeld and L. Mahadevan, Phys. Rev. Lett. 106, 105702 (2011).

[4] J. Dervaux, Y. Couder, M. A. Guedeau-Boudeville, and M. Ben Amar, Phys. Rev. Lett. 107, 018103 (2011).

[5] E. Hohlfeld and L. Mahadevan, Phys. Rev. Lett. 109, 025701 (2012).

[6] D. P. Holmes and A. J. Crosby, Phys. Rev. Lett. 105, 038303 (2010).

[7] J. D. Paulsen, E. Hohlfeld, H. King, J. Huang, Z. Qiu, T. P. Russell, N. Menon, D. Vella, and B. Davidovitch, Proc. Natl. Acad. Sci. U.S.A. 113, 1144 (2016).

[8] W. Hong, X. Zhao, and Z. Suo, Appl. Phys. Lett. 95, 111901 (2009).
[9] M. Ben Amar and P. Ciarletta, J. Mech. Phys. Solids 58, 935 (2010).

[10] H. Diamant and T. A. Witten, Phys. Rev. Lett. 107, 164302 (2011).

[11] S. Cai, D. Chen, Z. Suo, and R. C. Hayward, Soft Matter 8, 1301 (2012).

[12] Y. Cao and J. W. Hutchinson, Proc. R. Soc. A 468, 94 (2012).

[13] A. Ghatak and A. L. Das, Phys. Rev. Lett. 99, 076101 (2007).

[14] J. Dervaux and M. Ben Amar, Annu. Rev. Condens. Matter Phys. 3, 311 (2012).

[15] D. Chen, S. Cai, Z. Suo, and R. C. Hayward, Phys. Rev. Lett. 109, 038001 (2012).

[16] H. Wang and S. Cai, Soft Matter 11, 1058 (2015).

[17] L. Jin, D. Chen, D. Chen, Z. Suo, and R. C. Hayward, Soft Matter 10, 303 (2014).

[18] P. S. Stewart, S. L. Waters, T. E. Sayed, D. Vella, and A. Goriely, Extreme Mechanics Letters 8, 22 (2016).

[19] M. A. Biot, Appl. Sci. Res. 12, 168 (1963).

[20] See Supplemental Material at http://link.aps.org/ supplemental/10.1103/PhysRevLett.119.198001 for details on experimental and numerical methods.

[21] J. Eggers and M. A. Fontelos, Singularities: Formation, Structure, and Propagation (Cambridge University Press, Cambridge, England, 2015).

[22] J. Eggers and N. Suramlishvili, Eur. J. Mech. B 65, 107 (2017).

[23] J.-T. Jeong and H. K. Moffatt, J. Fluid Mech. 241, 1 (1992).

[24] E. Lorenceau, Ph.D. thesis, Université Pierre et Marie Curie-Paris VI, 2003, https://tel.archives-ouvertes.fr/tel00003275 .

[25] J. Eggers and M. A. Fontelos, Panoramas et Synthèses 38, 69 (2012)

[26] Z. Suo, Finite deformation: general theory (2013), http:// imechanica.org/node/538.

[27] Z. Suo, Elasticity of rubber-like materials (2013), http:// imechanica.org/node/14146.

[28] M. Heil and A. Hazel, in Fluid-Structure Interaction, edited by M. Schäfer and H.-J. Bungartz (Springer, New York, 2006), pp. 19-49.

[29] M. Singh and A. C. Pipkin, J. Appl. Math. Phys. 16, 706 (1965).

[30] K. L. Johnson, Contact Mechanics (Cambridge University Press, Cambridge, England, 1985). 